\title{
XI
}

\section{Galilean Particles: An Example of Constitution of Objects}

\author{
Elena Castellani
}

\section{Objects and Physics}

Speaking of physical objects, we can momentarily bracket the general question "What is there?" and focus on the more specific problem of what could reasonably be taken as an object in the context under examination.

As regards physics, it is uncontroversial that subatomic and subnuclear particles - the best candidates for qualifying as "objects" in the realm of contemporary physics - are quite different from the ordinary physical objects we can see or touch in our everyday experience. Microphysical entities are surely not immediate data of perception: they can be "observed" only with the help of instruments, and sometimes they are even in principle unobservable as free particles, as in the well known case of quarks. Moreover, properties traditionally attributed to an object, such as its persistence through time or the possibility of distinguishing it from another similar object, are not easily available in quantum contexts.

These are some of the reasons why it is often claimed that the classical conception of physical objects, that is, the conception which is grounded on everyday experience, is no longer appropriate when we turn to microphysics. Quantum objects are remote from the objects of our common understanding; if microparticles are to be taken as objects, this has to be justified: they must be constructed or "constituted" as objects, depending 
on the theoretical framework being used as well as on the experimental data at disposition.

Supporters of this view usually assume that, with regard to the characterization of objects of physics, difficulties typically arise in the case of non-classical entities; in classical physics, objects are not really different from ordinary "material beings."

But one can arrive at a more radical position. Physics, classical or not, does not speak immediately of the objects which populate our external world. Classical mechanics, for example, is formulated in terms of mass points, which are obviously of quite another nature than everyday things. Mass points can be seen as "ideal objects", taken to represent some main features of ordinary physical objects. ${ }^{1}$ How the properties symbolized through such ideal entities can actually be related to some "real" macroscopic object has to be clarified. And those same classical material beings, whose physical behaviour is described using the mass points representation, are not always directly given to us as definite objects: take the case of some astronomic bodies of which we have only a series of isolated light points as empirical evidence. ${ }^{2}$

Arguments of the kind above may lead to the following position: all the objects of physics, classical as well as not-classical, must be "constituted." 3 Such a position can be taken as the starting point of what we shall call the group-theoretical approach to the problem of physical objects. In this paper we shall give a brief overview of this approach, entering into some more details for the case of nonrelativistic objects, the so-called Galilean particles.

\section{Invariance, Symmetry Groups and Constitution of Objects}

In the framework of the "constitution view", objects are generally conceived as "carriers of properties." What the carriers themselves are is not a relevant point on this view. What matters is specifying which the constituting properties of an object are and how these properties are related to

\footnotetext{
${ }^{1}$ See for example W. V. O. Quine, Word and Object, Cambridge, Mass.: The M.I.T. Press, 1960, 248-251.

${ }^{2}$ This astrophysical example is treated, in particular, in P. Mittelstaedt, "The Constitution of Objects in Kant's Philosophy and in Modern Physics", in P. Parrini, ed., Kant and Contemporary Epistemology, Dordrecht: Kluwer Academic Publishers, 1994, which is reprinted above (Ch. X).

${ }^{3}$ The term constitution is here used to indicate that objects are determined as such by using some conceptual prescription - in the case of physics objects, some physical laws. For arguments supporting such an use of this "Kantian" terminology with regard to the problem of physical objects, see Mittelstaedt, "The Constitution of Objects", and also, of the same author, Sprache und Realität in der modernen Physik, Mannheim: Bibliographisches Institut-Wissenschaftsverlag, 1986.
} 
their carrier. The question is then: what is it that confers to the carrier of a set of properties the dignity of an object, or, more specifically, what kind of properties and prescriptions do we need in order to construct an object?

The group-theoretical approach to the problem of physical objects is grounded on the idea of invariance. More precisely, the basic consideration is that the fundamental role which the notions of invariance and symmetry (i.e., invariance with respect to a group of transformations) have acquired in contemporary physics can also provide a key for addressing the problem of the constitution of physical objects.

Arguments attributing special significance to the notion of invariance with regard to the object question are surely not new. Permanence or invariance in time is a classical requirement for defining the identity of an object in the philosophical tradition. More generally, invariance with respect to change in space and time, when interpreted as invariance under change of reference frames or "observers", is a typical objectivity condition required in determining physical objects: we would have difficulties in speaking of "objects" in the case of entities which were not recognizable as the same ones under a simple change of the spatio-temporal perspective.

What is specific to the group-theoretical approach for constituting objects is the exploitation of the invariance idea by using the results of the application of group theory in physics. The theory of groups of transformations and their representations constitutes the appropriate mathematical tool for investigating the consequences of the symmetry characteristics of physical systems, that is the characteristics which are usually formulated in terms of invariance principles. In physics, the significance of symmetry groups started to be realized around the middle 1920s (with the consequent introduction of group-theoretical techniques in theoretical elaboration) mainly thanks to the fundamental contributions of Hermann Weyl and Eugene Wigner on the application of group theory in quantum mechanics. Today, we can say that symmetry groups are among the basic ingredients of theoretical physics: the so-called "elementary particles" and their interactions are described essentially with the help of symmetry arguments and group-theoretical methods.

What can the theory of symmetry groups actually tell us in relation to the constitution of objects? Let us begin by recalling the well known work of Wigner (1939) on the representations of the inhomogeneous Lorentz group (also called "Poincaré group"), a locus classicus for the above approach to the object question. ${ }^{4}$ Wigner's aim was to determine all the unitary representations of Poincaré group (the space-time symmetry group

\footnotetext{
${ }^{4}$ E. Wigner, "On unitary representations of the inhomogeneous Lorentz group," $A n$ nals of Mathematics 40, No. 1 (1939): 149-204.
} 
of special relativity), investigating the connection of the representations with quantum mechanical wave equations. Among the far reaching results he obtained, we find the possibility of arriving at a complete classification of free relativistic elementary systems in association with the study of the irreducible representations of the Poincaré group. This possibility of establishing a correspondence between "irreducible representations" of the symmetry group and "elementary physical systems" furnishes the basic motivation for the group-theoretical approach to the constitution of objects. Since the fundamental work of Wigner, indeed, it has become quite usual to classify elementary particles on the basis of their correspondence with the irreducible representations of symmetry groups. In fact, with respect to the characterization of objects it is possible to use symmetry considerations in more than one way. Let us focus on the two main arguments we can find in the literature.

A first possibility is to say, following Wigner's 1939 results, that each "elementary particle" is associated with an irreducible representation of the symmetry group. ${ }^{5}$ This implies that the particle has a given number of invariant properties, and that these are exactly the properties which characterize the kind of particle in question. ${ }^{6}$ Note, however, that what we obtain in this way is no more than a class of objects. The invariant properties which we ascribe to a "particle" on the ground of group-theoretical considerations are not sufficient for constituting the particle as an individual object, distinct from other similar particles. These properties are "necessary" or "essential", in the sense that the given "particle-object" couldn't be determined as such without them (an electron couldn't be an electron without given values of mass and spin). But an object cannot be determined as an individual object just on the basis of such "essential" properties (also called, in the literature, "intrinsic" properties). One has therefore to deal with the well-known problem of how to obtain "individuating" properties, or properties which can confer individuality on the particle in question, distinguishing it from the other similar particles, that is, from the other particles having the same invariant "essential" properties.

Here comes to the rescue another kind of symmetry argument, which goes back to the notion of a system of imprimitivity associated with a symmetry group. The method of imprimitivity systems, which proved to be of

\footnotetext{
${ }^{5}$ More precisely, an elementary particle is described as a physical system whose states transform under the operations of the symmetry group according to a definite irreducible representation.

${ }^{6}$ In the context of relativistic quantum physics, for example, an elementary particle is associated with an irreducible representation of the Poincaré group: within such a determination, the invariant properties characterizing a (free) particle are its rest-mass and spin.
} 
great importance for the theory of group representations and the application of this theory to the domain of quantum physics, was systematically developed in a series of papers of G. W. Mackey in the 1950 's. ${ }^{7}$ In the literature, the explicit use of the notion of an imprimitivity system with regard to the definition of a "particle" is due especially to Piron. ${ }^{8}$ The basic idea is to obtain a definition of a particle by employing physical quantities or observables, such as for example the position observable, through which the particle could be determined also as an individual object. As we shall see, the method of Mackey's imprimitivity systems for the space-time symmetry group provides a way of approaching the object problem from this point of view.

As an example of the above ideas, let us now have a closer look at how "particles" can in fact be constituted in the case of nonrelativistic (classical and quantum) mechanics.

\section{Galilean Particles (1)}

The space-time symmetry group of nonrelativistic physics is the socalled Galilei group, the group of transformations relating classical frames of reference. According to the group-theoretical approach, for arriving at obtaining "Galilean particles" one has therefore to investigate the irreducible representations of this symmetry group. This has to be done in the formalism of either classical or quantum mechanics, depending on what kind of objects - classical or quantum objects - we want to obtain. But let us start with some general features for discussing physical systems, space-time symmetry and the consequences of this symmetry for the physical description.

A physical system, to begin with, is generally described by first specifying its observable properties or observables and its possible modes of preparation or states. Observables are physical quantities which are measurable attributes of the system, i.e., their values can be measured on the system. The result of such measurements depends on the conditions in which the system is, that is its modes of preparation or states. Given observables and states, the description of the system at a certain moment is then completed by assigning a rule which tells us the expected value or expectation value of every observable for every state of the system. A

\footnotetext{
${ }^{7}$ For a clear and detailed account of Mackey's contributes on the notion of an imprimitivity system and a discussion of the impressive variety of applications of the notion, see in particular the chapter on systems of imprimitivity and "Mackey's machine" in V. S. Varadarajan, Geometry of Quantum Theory, 2d ed., New York: Springer, 1985.

${ }^{8}$ See C. Piron, Foundations of Quantum Physics, Reading, Mass.: W. A. Benjamin, 1976, $93 \mathrm{ff}$. A definition of an "elementary particle" making use of the notion of a system of imprimitivity can be found already in J. M. Jauch, Foundations of Quantum Mechanics, Reading, Mass.: Addison-Wesley, 1968, 205 ff.
} 
"dynamical description" will be obtained by specifying, in addition to the above "instantaneous description", how the system evolves with time, that is, by furnishing its evolution law (or dynamical law).

Space-time symmetry is the symmetry postulated through the principle of relativity (the laws of physics are invariant under changes of reference frame). In group-theoretical terms, this means the invariance of physical laws with respect to the group of transformations of reference frames, i.e., the group of space-time symmetry consisting of the translations in space and time, the spatial rotations and the transitions to a uniformily moving coordinate system. In the case of so-called "nonrelativistic physics", ${ }^{9}$ this is precisely the group of Galilean space-time transformations or Galilei group.

Given a physical system and its description, what follows from the assumption of space-time symmetry? In general, if $G$ is a symmetry group of a theory describing some physical system (i.e., the fundamental equations of the theory are invariant under the transformations of group $G$ ) this implies, among other things, that the states of the system transform into each other according to some representation of the group: the group operations are "represented" in the states space by operations relating the states one to each other. ${ }^{10}$

Accordingly, if the Galilei group is the symmetry group of the theory, the set of the states of the system should provide a "representation space" for this group. We shall therefore proceed towards the determination of "Galilean particles" by first defining as a Galilean system a physical system whose states form a representation space for the Galilei group. ${ }^{11}$ In order to arrive at Galilean particles, the strategy will then be to select, among Galilean systems, those which are elementary. For a physical system representing a single particle, elementarity is a quite natural requirement already from an intuitive point of view. In group-theoretical terms, however, this assumes the following precise meaning: an elementary system is a system whose set of states constitutes a representation space for an $i r$ reducible representation of the space-time symmetry group. ${ }^{12}$ This means,

\footnotetext{
${ }^{9}$ Which it would be more appropriate to call "Galilean relativistic physics", as it has been particularly stressed by J.-M. Lévy-Leblond. See for instance his "Galilei Group and Galilean Invariance", in E. M. Loebl, ed., Group Theory and Its Applications, vol. 2. New York: Academic Press, 1971.

${ }^{10}$ Where the basic condition for a set of operations $T\left(g_{i}\right), T\left(g_{j}\right), \ldots$ to form a "representation" of the group $G$ (with elements $g_{i}, g_{j}, \ldots$ ) is that $T\left(g_{i}\right) T\left(g_{j}\right)=T\left(g_{i} g_{j}\right)$.

${ }^{11}$ In an analogous way, a Lorentz system will be defined as a system whose states form a representation space for the Lorentz group.

${ }^{12}$ About the fact that the concept of an "elementary system" is indeed broader than the intuitive concept of an "elementary particle" (an elementary particle requiring the further condition of being structureless), see in particular T. D. Newton and E. P. Wigner, "Localized States for Elementary Systems", Review of Modern Physics, 21 (1949): 400406.
} 
in other words, that there is a correspondence between the "elementarity" of the system and the "irreducibility" of the representation associated with the system: for such a system, it will not be possible that the set of its states decomposes into (linear) subsets which are each invariant under the transformations of the space-time symmetry group. ${ }^{13}$

At this point, we can propose the following definition of a Galilean particle:

Definition (1): a Galilean particle is an elementary Galilean system, i.e., a physical system whose states form an irreducible representation space for the Galilei group.

Actually, in order to take into account the fact that we have a whole class of equivalent elementary Galilean systems, we should more accurately say:

Definition (1'): a Galilean particle is a physical equivalence class of elementary Galilean systems.

What have we obtained so far? Let us see what the above definition implies. For this purpose, we shall have to return to a more abstract level and introduce some aspects of the Lie group formalism appropriate for investigating the irreducible representations of space-time symmetry groups.

Space-time transformation groups such as the invariance groups of Galilean and Lorentz relativity are continuous groups, and in particular Lie groups. This means, first of all, that the group elements are functions of a certain number $r$ of continuous parameters $a_{l}(l=1,2, \ldots r)$, which are characteristic of the group. Without entering into further mathematical technicalities, let us just recall that such group elements can be written in terms of a corresponding number $r$ of infinitesimal operators $X_{l}$, the generators of the group, which satisfy the "multiplication law" represented by the "Lie brackets"

$$
\left[X_{s}, X_{t}\right]=c_{s t}^{q} X_{q}
$$

so forming what is called the Lie algebra of the group. The coefficients $c_{s t}^{q}$ are constants which characterize the particular structure of the group, whence their being named the structure constants of the Lie group. For every Lie group, we can construct operators which are scalar quadratic in the infinitesimal operators $X_{l}$, the so-called Casimir operators

$$
C=\sum_{s, t}\left(g^{-1}\right)_{s t} X_{s} X_{t}
$$

\footnotetext{
${ }^{13}$ That is, subsets whose component states are transformed into states of the same subset by the transformations of the symmetry group.
} 
with the matrix elements $g_{s t}$ given by $g_{s t}=\sum_{p q} c_{s q}^{p} c_{t p}^{q}$. Casimir operators have the special property of commuting with all the infinitesimal operators $X_{l}$ (the generators of the Lie group), that is, they are the fundamental invariants of the group. In the context of the theory of group representations, this implies, in particular, that in an irreducible representation the Casimir operators are simple multiples of the unit operator, whence the possibility of labelling the representations directly in terms of the eigenvalues of these operators. The eigenvalue spectra of the invariants of the group therefore provide the labels for classifying the irreducible representations of the group: on this fact is grounded the possibility of associating the values of the invariant properties characterizing physical systems with the labels of the irreducible representations of symmetry groups.

This is a very general scheme, which can be abstracted from the usual way of proceeding in quantum relativistic physics (quantum field theory) for classifying elementary particles. In order to see how and to what extent such a scheme can be applied to the case of classical and quantum "Galilean particles", one has to consider how classical and quantum physical systems can respectively provide a representation space for irreducible representations of the Galilei (Lie) group, and what kind of invariant properties can be consequently attributed to either class of systems. In this paper we shall give only a very brief account of this program, by summarizing it in the following points. ${ }^{14}$

\subsection{Galilei Group}

The (proper) Galilei group $\mathcal{G}$ is a 10 -parameter Lie group, containing the translations in space $\mathbf{x}^{\prime}=\mathbf{x}+\mathbf{a}(3$ translation parameters $\mathbf{a})$, the translations in time $t^{\prime}=t+b$ ( time parameter $b$ ), the space rotations $\mathbf{x}^{\prime}=R \mathbf{x}$ (3 rotation parameters in rotation matrix $R$ ), and the transitions to a uniformly moving coordinate system $\mathbf{x}^{\prime}=\mathbf{x}+\mathbf{v} t$, also called "pure Galilei transformations" or "Galilei boosts" (3 velocity parameters $\mathbf{v})$.

A general inhomogeneous Galilei transformation is then a map

$$
g:(\mathbf{x}, t) \mapsto\left(\mathbf{x}^{\prime}, t^{\prime}\right)
$$

of the form

$$
\mathbf{x}^{\prime}=R \mathbf{x}+\mathbf{v} t+\mathbf{a}
$$

\footnotetext{
${ }^{14}$ For simplicity sake, in considering the states of a physical system we shall take into account only the case of "pure state." (A more general treatment of representation spaces for the space-time symmetry group, which includes also the case of "mixed states", is of course possible; see, for example, D. G. Currie, T. F. Jordan, and E. C. G. Sudarshan, "Relativistic Invariance and Hamiltonian Theories of Interacting Particles", Review of Modern Physics 35 (1963): 350-375.)
} 


$$
t^{\prime}=t+b
$$

In terms of the group parameters, a generic element $g$ of $\mathcal{G}$ is denoted by

$$
g=(b, \mathbf{a}, \mathbf{v}, R)
$$

and the multiplication law of $\mathcal{G}$ is

$$
g g^{\prime}=(b, \mathbf{a}, \mathbf{v}, R)\left(b^{\prime}, \mathbf{a}^{\prime}, \mathbf{v}^{\prime}, R^{\prime}\right)=\left(b+b^{\prime}, \mathbf{a}+R \mathbf{a}^{\prime}+b^{\prime} \mathbf{v}, \mathbf{v}+R \mathbf{v}^{\prime}, R R^{\prime}\right) .
$$

Given the multiplication law or "group law" and hence the group structure, ${ }^{15}$ one can compute the Lie brackets for the infinitesimal generators and consequently obtain the Lie algebra of the Galilei group $\mathcal{G}$.

\subsection{Galilei Group and Classical Mechanics}

The usual way of describing a classical mechanical system with $n$ degrees of freedom is to associate with it a space $\Omega$, called the phase space of the system, whose points are defined by the values $x_{1}, \ldots, x_{n}, p_{1}, \ldots, p_{n}$ of the configuration $\left(x_{1}, \ldots, x_{n}\right)$ and the momentum vector $\left(p_{1}, \ldots, p_{n}\right)$ of the system at a given instant of time. These points represent the states of the system and the physical quantities or observables are described by real valued functions defined on the phase space. The system we are considering here is a massive point particle.

In the phase space of the states of the system, the symmetry transformations are "represented" by so-called canonical transformations, which are the transformations of the phase space leaving invariant the equations of motion. Canonical transformations can be regarded as infinite sequences of infinitesimal canonical transformations $x_{i}^{\prime}=x_{i}+\delta x_{i}, p_{i}^{\prime}=p_{i}+\delta p_{i}$. The quantities $W_{s}$, such that $\delta x_{i}=\left(\partial W_{s} / \partial p_{i}\right) \delta \alpha_{s}$ and $\delta p_{i}=-\left(\partial W_{s} / \partial x_{i}\right) \delta \alpha_{s}$, are called the generators of the infinitesimal transformations.

The Lie algebra of the Galilei group has a physically significant realization in terms of the ten quantities $H, \mathbf{P}, \mathbf{J}$, and $\mathbf{K}$, which are the generators of the canonical transformations representing the time translations, space translations, space rotations and Galilean boosts, respectively. $H$ can be identified with the Hamiltonian or energy of the system, the three generators $P_{i}$ with the components of the momentum $\mathbf{P}$, the three generators $J_{i}$ with the components of the angular momentum $\mathbf{J}$ and the three generators $K_{i}$ with the components of the quantity $\mathbf{K}=t \mathbf{P}-m \mathbf{X}$. The Lie brackets characterizing the Lie algebra of the Galilei group are represented by the

\footnotetext{
${ }^{15}$ The multiplication law is easily obtained by applying successively two Galilei transformations $g$ and $g^{\prime}$.
} 
following Poisson brackets:

$$
\begin{aligned}
& \left\{P_{i}, P_{j}\right\}=0, \quad\left\{P_{i}, H\right\}=0, \quad\left\{J_{i}, H\right\}=0, \\
& \left\{J_{i}, J_{j}\right\}=\epsilon_{i j k} J_{k}, \quad\left\{J_{i}, P_{j}\right\}=\epsilon_{i j k} P_{k}, \\
& \left\{J_{i}, K_{j}\right\}=\epsilon_{i j k} K_{k}, \quad\left\{K_{i}, H\right\}=P_{i}, \quad\left\{K_{i}, K_{j}\right\}=0,
\end{aligned}
$$

and

$$
\left\{K_{i}, P_{j}\right\}=0+m \delta_{i j}
$$

where the constant $m$ can be identified with the mass of the system. ${ }^{16}$

\subsection{Galilei Group and Quantum Mechanics}

In the usual formulation of quantum mechanics, the behaviour of a system with $n$ degrees of freedom is described in terms of a wave function or state vector $\psi(\mathbf{x}, t)$, where $\mathbf{x}=\left(x_{1}, \ldots, x_{n}\right)$ is the configuration of the system at the instant of time t. To each physical system is associated a Hilbert space $\mathcal{H}$, which is the space of the wave functions or vectors corresponding to the (pure) states of the system, and to each observable quantity corresponds a self-adjoint operator acting in this Hilbert space.

Any group $G$ of coordinate transformations $(\mathbf{x}, t) \mapsto\left(\mathbf{x}^{\prime}, t^{\prime}\right)$ defines a corresponding set of induced trasformations $\psi \mapsto \psi^{\prime}$ in the Hilbert space of the wave functions. If $G$ is a symmetry group of the system description, the transformations of the group are represented in the space $\mathcal{H}$ by unitary operators acting on the wave functions. In the case of a Lie group, the unitary operators can be regarded as generated by infinitesimal (self-adjoint) operators.

A physically significant representation of the Galilei Lie group is given in terms of the infinitesimal generators which are the quantum-mechanical analogues of the classical quantities generating the Galilean transformations: that is, the operator Hamiltonian $H$ for time translations, the three operators components of the momentum $\mathbf{P}$ for space translations, the three operators components of the angular momentum $\mathbf{J}$ for space rotations and the three operators components of the quantity $\mathbf{K}$ for Galilei boosts. The Lie brackets of the Galilei Lie algebra are here represented by the commutation relations

$$
\left[P_{i}, P_{j}\right]=0, \quad\left[P_{i}, H\right]=0, \quad\left[J_{i}, H\right]=0,
$$

${ }^{16}$ The generators $H, \mathbf{P}, \mathbf{J}, \mathbf{K}$ so constitute a "Poisson algebra" of the form

$$
\left\{W_{i}, W_{j}\right\}=c_{i j}^{k} W_{k}+\beta_{i j}
$$

(where $\beta_{i j}$ are constants), that is a so-called projective realization of the Galilei group Lie algebra in the phase space. 


$$
\begin{aligned}
& {\left[J_{i}, J_{j}\right]=\epsilon_{i j k} J_{k}, \quad\left[J_{i}, P_{j}\right]=\epsilon_{i j k} P_{k},} \\
& {\left[J_{i}, K_{j}\right]=\epsilon_{i j k} K_{k}, \quad\left[K_{i}, H\right]=P_{i}, \quad\left[K_{i}, K_{j}\right]=0,}
\end{aligned}
$$

and

$$
\left[K_{i}, P_{j}\right]=0+m \delta_{i j} I
$$

where $m$ is the mass of the system. ${ }^{17}$

\subsection{Invariant Properties}

As we have seen, the values of the fundamental invariants of the symmetry group label the irreducible representations and hence the elementary physical systems.

Both in the classical and quantum case, the characteristic invariant quantities one obtain by constructing the irreducible (projective) representations of the Galilei group are the mass $m$ (as can be seen also from the relations representing the Lie brackets for the Galilei group generators), the "internal energy" (i.e., the energy in the rest system) and a quantity which can be interpreted as an "internal angular momentum" or "spin." 18

From the group-theoretical point of view, "Galilean particles" are therefore characterized in terms of the invariant properties of mass, internal energy and spin. ${ }^{19}$

\section{Galilean Particles (2)}

As already remarked, another way of addressing the object question from the group-theoretical point of view is that grounded on the notion of a system of imprimitivity for the space-time symmetry group. The aim is to arrive a definition of a particle by determining "individuating" observable quantities (such as, for example, the position and momentum) with the help of the method of imprimitivity systems. ${ }^{20}$

\footnotetext{
${ }^{17}$ The infinitesimal operators $H, \mathbf{P}, \mathbf{J}, \mathbf{K}$ so form an algebra$$
\left[W_{i}, W_{j}\right]=c_{i j}^{k} W_{k}+\beta_{i j} I
$$

which is a projective representation of the Galilei group Lie algebra in the Hilbert space.

${ }^{18}$ For more details on these invariant quantities and their meaning in the classical case and in the quantum nonrelativistic case-and, in general, for what regards the Galilei group and its irreducible representations - see in particular Lévy-Leblond, "Galilei Group and Galilean Invariance", and also, by the same author, "Galilei Group and Nonrelativistic Quantum Mechanics", Journal of Mathematical Physics 4 (1963): 776788 .

${ }^{19}$ Recalling, however, that for a nonrelativistic isolated particle the internal energy has indeed no physical significance. See Lévy-Leblond, "Gal. Group and Nonrelat. Quant. Mechanics", 782.

${ }^{20}$ The view at issue here is that according to which individuality is conferred upon an object by some of its properties, and in particular by space-time properties. 
The framework is that of the logical formulation of a physical system: according to the viewpoint introduced in the 1930's by John von Neumann, to each physical system is associated a (orthocomplemented) lattice $\mathcal{L}$ of "propositions", which is called the logic of the system. These propositions are the most general experimentally verifiable statements which can be made about the system, that is the statements of the form "the value of a certain observable quantity lies in a given set of real numbers." In such a framework, if $A\{E\}$ is the proposition that, for a given system $S$, the value $a$ of the observable quantity $A$ lies in the set $E \in \mathcal{B}(\mathcal{R})$ (where $\mathcal{B}(\mathcal{R}$ ) is the family of the Borel subsets of the real line $\mathcal{R}$ ), the observable $A$ may be identified with the mapping

$$
A: E \mapsto A\{E\}
$$

of $\mathcal{B}(\mathcal{R})$ into the lattice $\mathcal{L}$.

Given the triplet $(A, \mathcal{B}, \mathcal{L})$ for a physical system, the method is then to consider what the action of the space-time symmetry group $G$ implies for the elements of the triplet. To state it very shortly, we shall have, in particular, a condition of covariance for the observable $A$, which can be expressed in terms of the following imprimitivity condition:

$$
A\{\sigma(g)[\mathcal{B}]\}=S(g)[A\{E\}]
$$

Graphically, the above condition corresponds to the commutativity of the diagram

$$
\{\text { Insertfigure } 1\}
$$

where $\sigma(g)$ and $S(g)$ are representations of the group $G$ in terms of the automorphisms of $\mathcal{B}$ and of $\mathcal{L}$, respectively. A triplet $(A, \mathcal{B}, \mathcal{L})$ which satisfy the above condition is called a system of imprimitivity for the group $G .^{21}$

Now, if one takes as "identifying" properties the observables position, momentum and time, a "Galilean particle" can be defined in the following way: ${ }^{22}$

Definition (2): a Galilean particle is an elementary system for which the observables position, momentum and time are defined.

that is, in the above described framework,

Definition (2'): a Galilean particle is a system of propositions $\mathcal{L}$, for which an irreducible representation of the Galilei group

\footnotetext{
${ }^{21}$ See for example Piron, Found. of Quant. Physics, 94-95.

${ }^{22}$ We follow here the approach of Piron.
} 
is defined such that the observable quantities $A_{i}: \mathcal{B}(\mathcal{R}) \mapsto \mathcal{L}$, where $i=1,2,3$ and $A_{1}, A_{2}, A_{3}$ are, respectively, the position, momentum and time, satisfy the imprimitivity condition $(*)$.

Galilean particles can then be explicitly constructed by studying the imprimitivity systems for the observables position, momentum and time both in the classical and in the quantum case. ${ }^{23}$

\footnotetext{
${ }^{23}$ Some examples of so constructed classical and quantum Galilean particles can be found in Piron, Found. of Quant. Physics, $97 \mathrm{ff}$. In the quantum case, the problem of the incommensurability of properties such as position and momentum can be approached by using so-called "unsharp observables." The problem of the (approximate) constitution of objects by means of unsharp properties, that is, by means of the study of imprimitivity systems in the case of unsharp observables, is investigated in particular by P. Mittelstaedt, P. Busch, and P. Lahti. On the subject see, for instance, Mittelstaedt, "The Constitution of Objects"; P. Busch, "Unsharp Reality and the Question of Quantum Systems", in P. Lahti and P. Mittelstaedt, eds., Symposium on the Foundations of Modern Physics 1987, Singapore: World Scientific, 1987, and P. Busch, "Macroscopic Quantum Systems and the Objectification Problem", in P. Lahti and P. Mittelstaedt, eds., Symposium on the Foundations of Modern Physics 1990, Singapore: World Scientific, 1990
} 